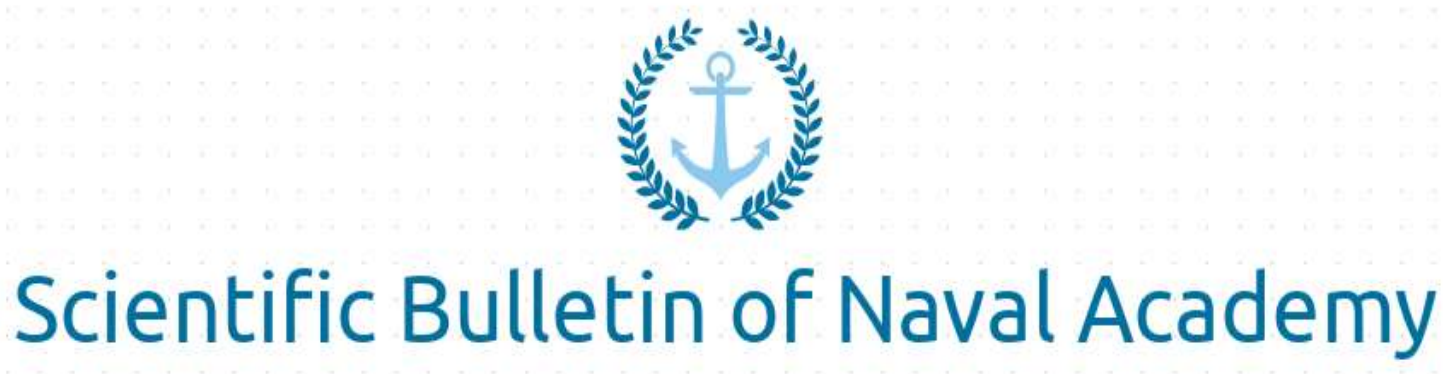

SBNA PAPER • OPEN ACCESS

A Review over Al Methods Developed for Maritime Awareness Systems

To cite this article: A Pohonțu, Scientific Bulletin of Naval Academy, Vol. XXIII 2020, pg.287-299.

Available online at www.anmb.ro

ISSN: 2392-8956; ISSN-L: 1454-864X 


\title{
A Review over AI Methods Developed for Maritime Awareness Systems
}

\author{
A POHONȚU \\ RO Navy Officer, Constanța, Romania \\ E-mail: pohontualexandru@gmail.com
}

\begin{abstract}
Due to their operations against illegal activities, maritime threats or collision prevention analysis, maritime surveillance plays a vital role in maritime traffic security and safety management. Today's maritime surveillance and awareness systems can integrate multiple data sources like: coastal, HFSWR and SAR radars, AIS or satellite imagery; and this process produces massive amounts of data. That available data can be processed, with the use of Artificial Intelligence (AI) methods and algorithms, to automatically monitor the maritime traffic and its implications in safety, security, economy and environment. This paper's purpose is to briefly reveal current AI techniques that have been researched and deployed in the industry, and to seize the opportunity of implementing them.
\end{abstract}

\section{Introduction}

Waters cover more than two-thirds of the Earth's surface and over $90 \%$ of all international trades are being done over the sea. This field has become the scenario of vast activities which have an important impact on security, safety, economy and environment. Their great extension hinders their monitoring, implying the surveillance of coastal and open sea areas by various Maritime Domain Awareness (MDA) Agencies [1].

The principal goals of an MDA include "enhancing transparency in the maritime domain to detect, deter and defeat threats" and "enable accurate, dynamic, and confident decisions and responses to the full spectrum of maritime threats" [2]. Maritime threats include nation-wide piracy and terrorism actions, but also environmental and social problematics [3]. One key challenge in maritime surveillance field is to automatically acquire the monitoring, analysis and understanding of these activities at sea.

Normally, an MDA system obtain its data from various types of sensors that are capable of quantifying the location, velocity and trajectory of multiple vessels (see Figure 1) in a designated area [4]. There are multiple types of sensors that provide heterogeneous data regarding maritime activities and the majority of that data that is used by Maritime Surveillance (MS) systems. Data integration techniques have widely been deployed in the MS domain and they imply the fusion of multiple sensors like: Automatic Identification System (AIS) transponders, Infrared (IR) camera, Synthetic Aperture Radar (SAR), Vessel Traffic Service (VTS) radar, High Frequency Surface Wave Radar (HFSWR), Over The Horizon (OTH) radar and microwave radar [6]. Combined and processed together with a large spectrum of contextual information, data can be used to monitor naval traffic and to discover activities that may involve threats regarding to maritime security and safety, illegal trafficking or eventual risks regarding environments, resources and navigation mobility [8]. 


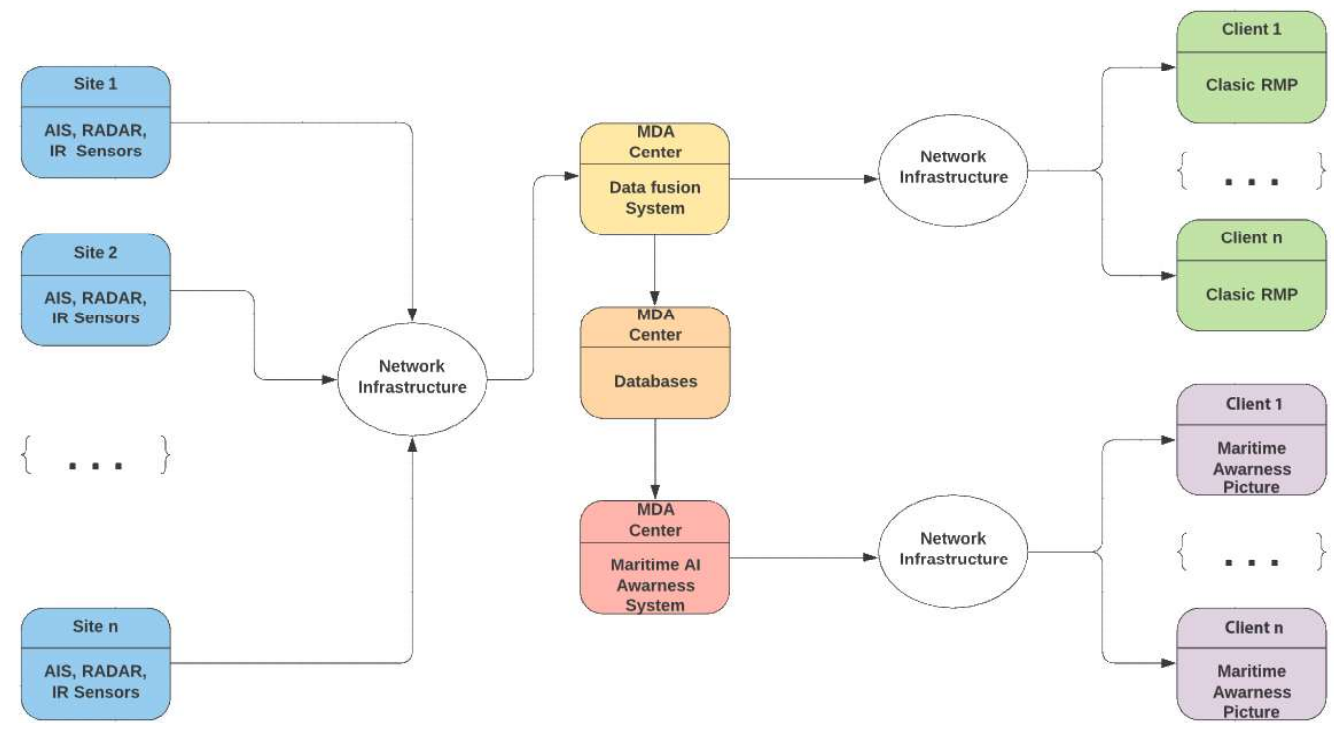

Figure 1. Example of a mixed MDA System

Even in today's continuous digitization context, maritime traffic management decisions are still being taken, in the majority of cases, on behalf of human experience. Monitoring open sea and harbours activities in order to detect suspicious behaviours represents a highly sophisticated task for the operators that keep track of the vessel movements.

Nowadays operator's analysis can be informed and extend by intelligent automated systems. Naval traffic keeps expanding, and the implications of using the maritime routes as a transportation highway has led to a demand for more advanced systems for monitoring and analysing maritime transportation spectrum. Most of what happens in the maritime domain is laborious to be observed and keep track of, with respect to vessel mobility, activities, and intentions. It is a necessity to use, in the best possible ways, all available data in order to detect and monitor suspicious behaviours [4]. Classical maritime surveillance systems are starting to get upgrades that include disruptive features coming from big data manipulation and machine learning. Present networks carry out a great amount of worldwide maritime traffic data that includes motion parameters as well as other significant attributes of vessels. The analysing process of this data with machine learning and big data techniques can provide an enhanced decision support for maritime domain [9]. The goal is to automatize the data acquisition, integration and interpretation processes, to offer the possibility to survey over large marine areas and to detect suspicious activities with reduced human interventions [1].

\section{Overview of Maritime Anomaly Detection}

The problematic of anomaly detection is a generic one, not tied especially to the maritime domain, and it occurs in many other fields. Data acquisition can be viewed as the first process that provides the raw input for anomaly detection. Typical behaviour models and, consequently, anomalous behaviours are then extracted from that data. The problem of data overload is a major reason for the evolution of anomaly detection techniques, which role is to find and underline information worthy of interest.

As mentioned before, anomaly detection is a complex process. It should ensure provide operators with relevant situational awareness information and support them in decision-making processes. Within the problem of anomaly detection in maritime traffic domain, two main types of approaches emerge, one focusing on defining anomalous behaviour, the other on seizing exceptions from the modelled normal behaviour. In the former case, the definition of anomalous behaviour is often defined based on expert knowledge and contextual data, and then used as a base for defining anomaly recognition and detection logic. In the latter case, the normal behaviour is captured in form of a statistical model, and deviations from it are marked as anomalous. It is efficient to combine the aid of 
human expertise with computing techniques. Machines are better suitable for operations that require processing large quantities of data while the human reasoning capacity is superior to that of the computers [4].

One major challenge into developing an intelligent MDA system pose the ability to identify patterns emerging within large amounts of data, combined from multiple sources and obtained from the monitoring process of numerous vessels. The derivation of implicit and often raw information from large databases is affiliated to the field of data mining. This is a multidisciplinary domain, which is linked to a variety of fields including statistics, database technologies, artificial intelligence or machine learning. Specifically, machine learning techniques provide the computational basis for data mining; its role is to extract relevant information from raw data and to infer underlying structures [3], [5].

In the following chapters there are listed and briefly explained the principal AI techniques, studied for identifying patterns in MDA Systems, based on two different approaches.

\section{3. "Exception from the Modelled Normal Behaviour" Approach}

One way for creating a standard model to seize normal vessel's behaviour is to cluster available data around a set of points in a multi-dimensional feature space. This feature can be positional data (longitude and latitude), velocity, trajectory or vessel's type [3]. Tracks that are within or near one of these clusters centroids are interpreted as normal, while the remainder are marked as potential anomalies [10].

The "Exception from the Modelled Normal Behaviour" Approach usually is used to build models of normal vessel behaviour from historical motion data. These models are then used to classify new vessel observations as normal or anomalous. They generally estimate the degree of deviation of new target from the learned model of normal positioning/location, speed or trajectories. In anomaly detection for MDA systems, algorithms are divided into two classes based on the models' learning characteristics: Geographical (map-dependent) model-based methods and parametrical (mapindependent) model-based methods [11].

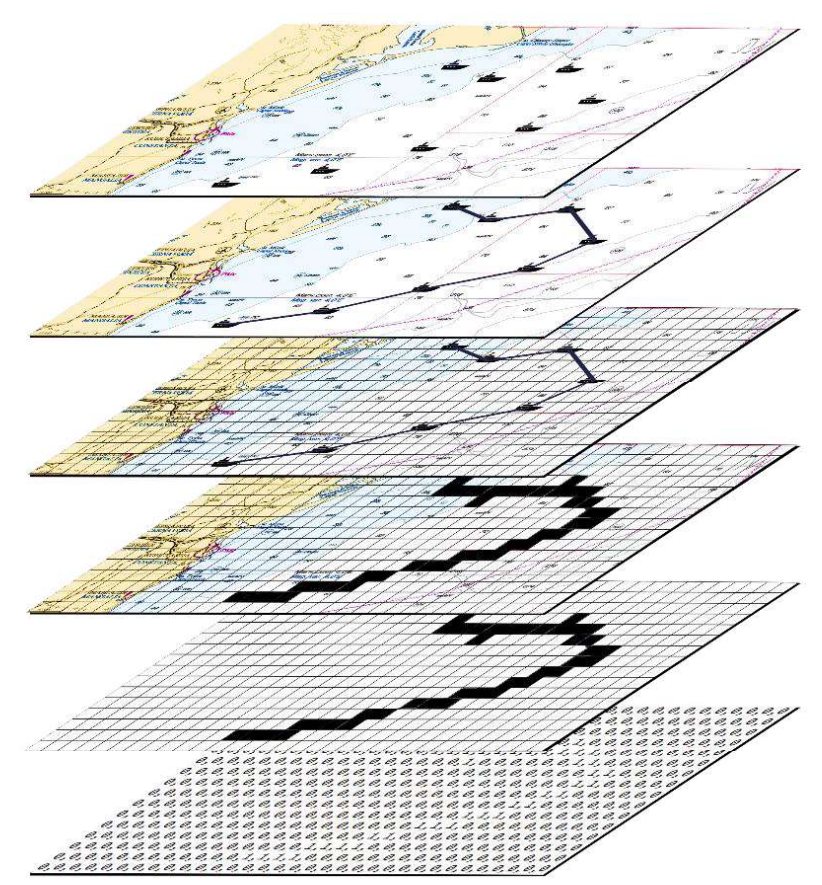

Figure 2. Geographical Grid Base Analysis Process of a Vessel Trajectory 
Maritime surveillance operators can be assisted into automatically recognition of relevant traffic situations by visualizing potential fields using modern rendering and clustering techniques. The first step of the proposed method is to assign charges to all passed locations for the geographical traces of vessel movements.

In a grid-based system, the strength of a charge $\mathbf{c}$ represents a metric obtained from sensor's data. Each discovered vessel is described by a collection of $\mathbf{n}$ numerical and non-numerical properties including vessel's static parameters (e.g., identification number, call sign, nationality), as well as the current state of its motion parameters (e.g., speed, drift, course).

$$
c_{\text {lat }_{k} \operatorname{lon}_{k}}=<c_{\text {lat }_{k} \operatorname{lon}_{k}}^{1}, c_{\text {lat }_{k} \operatorname{lon}_{k}}^{2}, \ldots, c_{\text {lat }_{k} \operatorname{lon}_{k}}^{n}>
$$

\subsection{Gaussian Distribution of Spatial Positions Method}

According to [4], Maritime Spatial Planning (MSP) is the process of representing, both spatially and temporally, the allocation plan distribution of naval activities. Vessel's navigation patterns can be viewed by constructing and analysing the routes from raw sensor's data.

In a grid-based model, the monitored area is divided into cells to create a spatial grid. The cells are then filled with motion properties of the crossing vessels [12] (see Figure 2).

The total charge $\mathbf{C}$ at a location $\mathbf{k}$ is counted as the sum of all local charges $\mathbf{c}$ accumulated over a time period $\tau$ :

$$
C_{\text {lat }_{k} \operatorname{lon}_{k}}=\sum_{t=0}^{\tau} C_{\text {lat }_{k} \operatorname{lon}_{k}}
$$

The potential field of a single charge reaches its maximum value at the location of the charge, and it reduces within a radius around it. Areas, where potential field has a high value, are considered a normal traffic patterns and areas where a potential field has a low value, signalize potential anomalous behaviour. The total potential at location $\mathbf{k}$ is the superposed potential generated by all surrounding charges in locations $\mathbf{i}$, decreased by the distance between these locations. Here the potential distribution $\mathbf{P}$ is described by two-dimensional Gaussian smoothing, using Euclidean distance for measuring the radius between two points:

$$
P_{\text {lat }_{k} \operatorname{lon}_{k}}=\sum_{i} \frac{1}{2 \pi \sigma} e^{\frac{\left(\operatorname{lat}_{k}-\text { lat }_{i}\right)^{2}+\left[a\left(\operatorname{lon}_{k}-\operatorname{lon}_{i}\right)\right]^{2}}{2 \sigma^{2}}}
$$

where $\mathbf{a}$ is a constant multiplier compensating the proportional difference between the Euclidean distance per a unit of longitude versus a unit of latitude, and the standard deviation $\boldsymbol{\sigma}$ is assumed same for both dimensions:

$$
C_{\text {lat }_{k} \operatorname{lon}_{k}}(t)=\sum_{t=0}^{\tau} d(t) c_{\text {lat }_{k} \text { lon }_{k}}
$$

where $\mathbf{d}(\mathbf{t})$ is a non-increasing decay function with limit at zero, describing the decrease of a local charge over time [4]. 


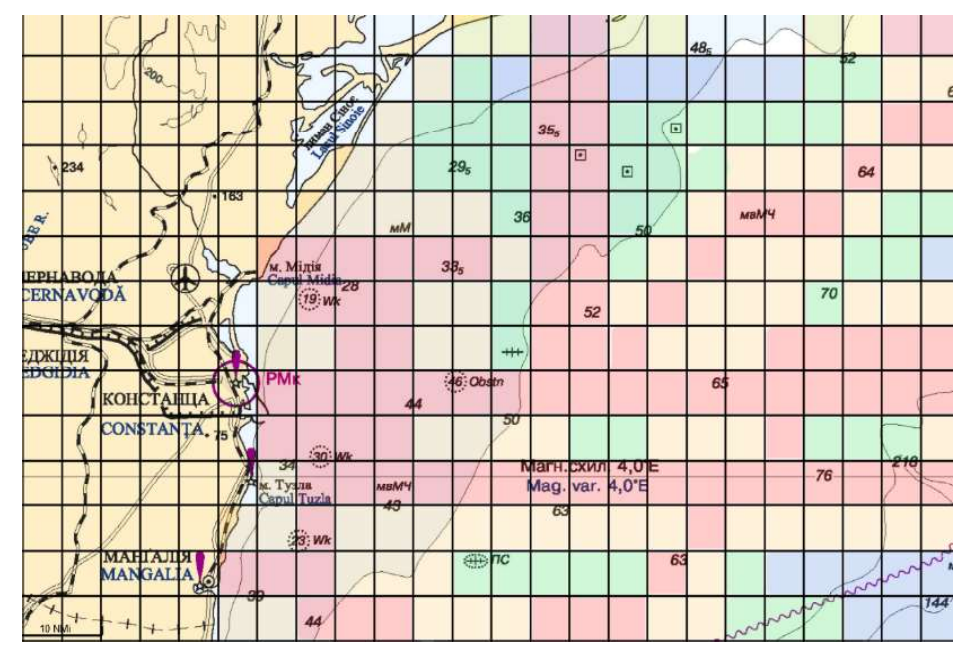

Figure 3. Gaussian Traffic Distribution (3x4 Nm Grid Size Simulation)

In bottom-up approaches, Grid-based methods divide regions of interest into cells and discretize local information such as velocity and location (see Figure 3). These methods make use of learning methods such as information theory, Hebbian learning and Hidden Markov models (HMMs). These methods have widely been used because of their ease of implementation and visualization but some drawbacks of them are their low accuracy and resolution problematic for the determining the grid size. HMMs can also be implemented with other techniques such as clustering to categorize different ship's tracks types in a hierarchical way [12]. Also, in studies like [13], there are used Gaussian Processes (GPs) for the extraction of features, and distance measures like Hellinger distance for grouping. The main advantage of GPs is their flexible and nonparametric nature that can be used to avoid problems like estimation of parameters such as the size-of-grid in grid-based methods, or Gaussians number in Gaussian Mixture Models (GMMs), as used in [13], [14].

\subsection{Anomalous Routes Pattern Recognition Method}

Developing data analytical methods for vessels behaviour can imply tasks of modelling that are capable of making statements related to their present and future state. Groups of vessels with "normal" patterns can be used to identify potential deviations from these due to possible suspicious conditions. This type of information can pose a great relevance since abnormal trajectories tend to display early indicators of potential problems, which are worth of attention and need to be solved in a quick manner. As such, in the domain of automated maritime surveillance, there are numerous applications that require real-time monitoring of abnormal sequential patterns over recorded trajectories [15].

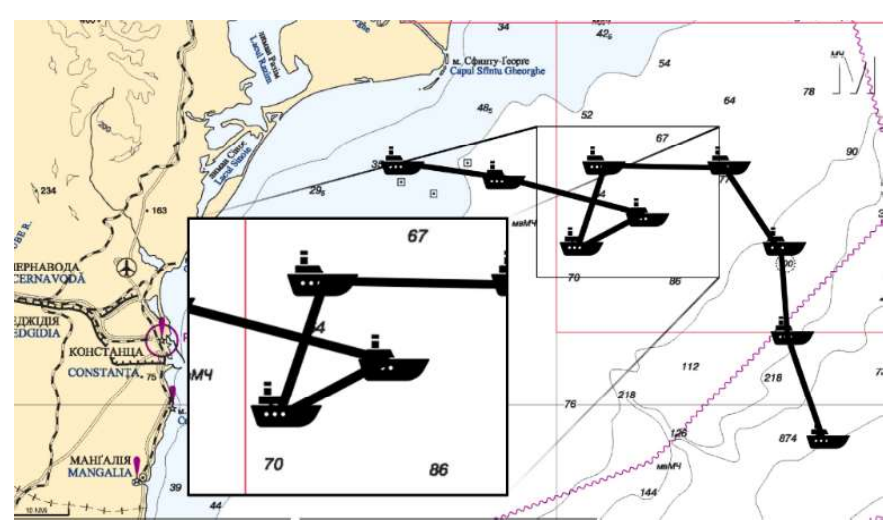

Figure 4. Anomalous Ship Trajectory Simulation 
Normally, vessels tend to perform constant changes in their heading for maintaining a predefined planned route, so they rarely present significant course over ground (COG) variations.

Several techniques based on spatio-temporal data mining methods have been developed to detect and construct vessels motion patterns inherent in maritime traffic raw data. In [16] and [17], it is presented a method for monitoring fishing vessels by clustering them with the use of spatial data information. The author investigates a method for extracting vessel traffic patterns that could identify high-risk tracks by analysing coastal AIS data. His proposed method extracts routes using densitybased clustering and generalizes vessels turns using angle thresholds techniques.

Another method based on an adapted version of the Shared Nearest Neighbour algorithm is used in [18] to cluster vessels moving towards similar directions. The developed clustering process analyses the vessels positions and directions in order to identify maritime traffic routes.

In [19], a vectorial approach is introduced. Here, trajectories are represented as a set of lines connecting multiple nodes. The authors proposed this method to automatically detect trajectories and cluster them together into routes. In [20], the same method was used to detect routes as initial structures of the maritime traffic, and after that the authors proposed a method to generalize and merge the routes in order to build networks capable to represent maritime traffic.

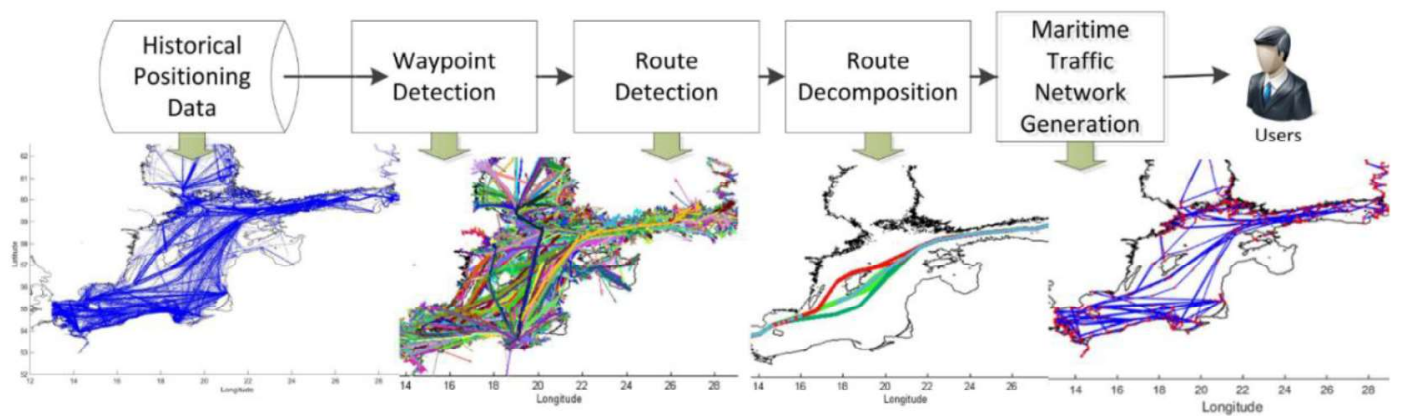

Figure 5. Route extraction technique [1]

In [1], the authors present an unsupervised approach for generating maritime traffic representation from historical self-reporting positioning data based on spatio-temporal data analysis techniques. The advantage of this proposed representation, is that it provides a light, structured and precise representation.

\subsection{Velocity Variations Analysis Method}

Another extensively applied technique in maritime traffic studies are statistics-based approaches. Traffic statistics can provide both quantitative modelling and representation of the traffic features. It can be used to determine relevant traffic parameters and thresholds in order to help constructing normal vessels behaviours. A common statistics-based representation of maritime traffic speed patterns is illustrated in Figure 6. 


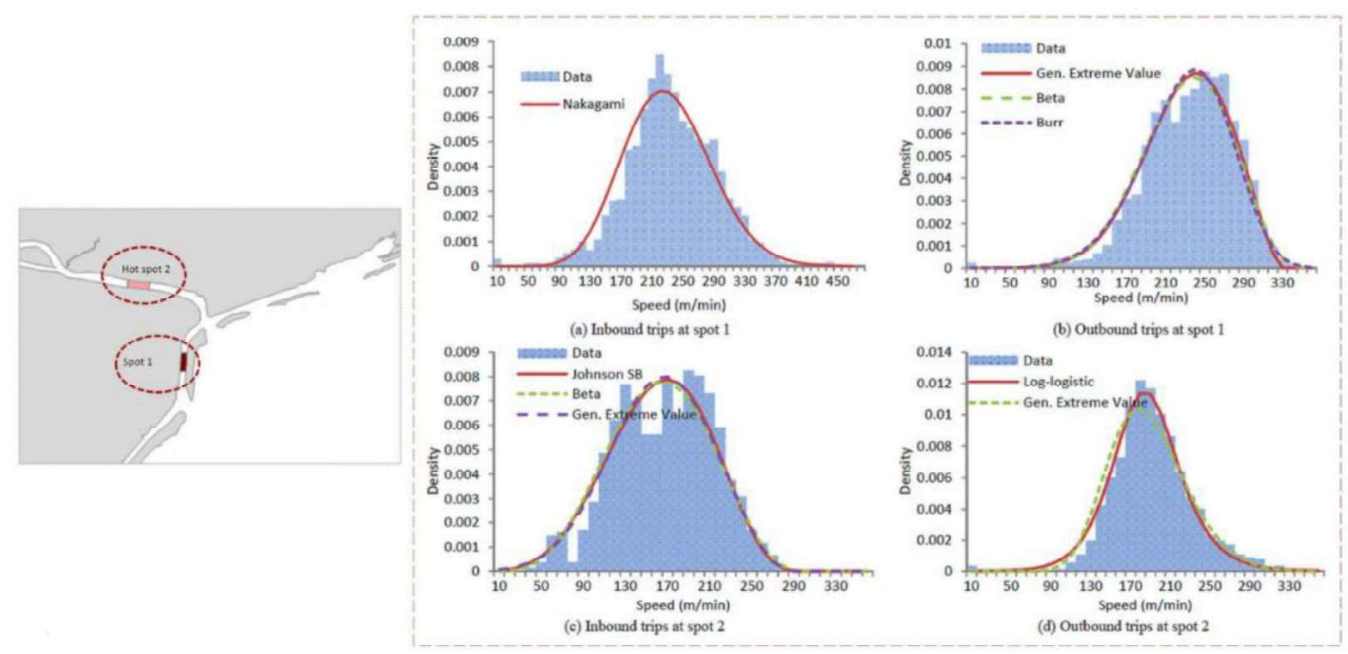

Figure 6. Anomalous Ship Speed Variation Graphs [41]

In [21], the authors propose a statistical model of vessel motion patterns using adaptive kernel density estimation (KDE). It is used to describe the distribution of location and velocity vectors from AIS raw data [21]. The established KDE model can detect traffic anomalies through quantilebased thresholds and differentiate the anomalous behaviour from normal one. The motion patterns can also be applied to support maritime traffic prediction. It is mainly based on a particle filtering process with "nearly constant velocity motion model".

In [22], the author presents a spatio-temporal mining algorithm named LOWESS (locally weighted scatterplot smoothing). It adopts nonparametric regression, to obtain a nonlinear curve to fit the trajectory point clouds. In contrast to another vastly deployed algorithm, named DBSCAN (Density-based spatial clustering of applications with noise), rather than clustering the route trajectory from the mass of points, this LOWESS uses nonparametric regression to establish the trajectory model by fitting the point clouds historically collected [23].

\subsection{Neural Networks Based Method}

Neural networks represent a small part of the artificial intelligence (AI) domain. They can carry out very small and highly specific tasks and pattern recognition is one task that neural networks were designed for [24].

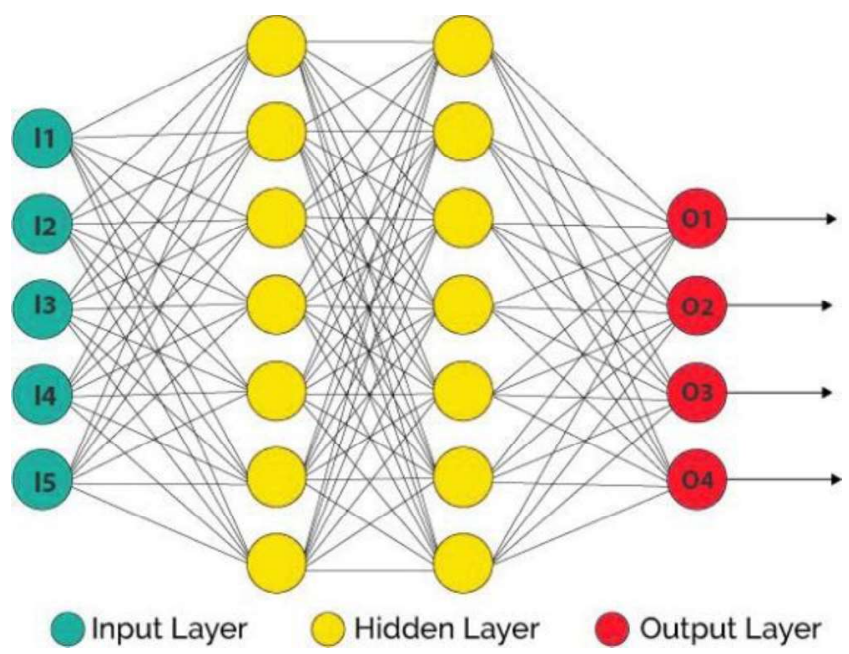

Figure 7. Deep Neural Network Model 
An artificial neural network (ANN) is made of a collection of connected nodes called neurons, similar to the neurons in a biological brain (see Figure 7). Each connection, like the biological brain's synapses, transmit a signal between neurons. An artificial neuron normally receives a signal, processes it and after that signals other neurons connected to it. A connection's signal is represented in the form of a real number, and the output of each neuron is computed by non-linear function that has one or multiple inputs. These connections are defined as edges. Neurons and edges typically have different weights that are adjusted in the time of learning process [42].

In recent years, neural networks have intensively been applied for computing vessels' behavioural state, position and trajectory predictions. In several works, researchers documented the viability of adapting different types of neural networks like generic Artificial Neural Network (ANN) [25], [26], [27], neuro-evolution ANN [165], Back-Propagation (BP) network [28] or Generalized Regression Neural Network (GRNN) [29] for vessels prediction task.

From a training perspective view, two ways to build a neural network-based model emerge: "onetime training procedure" and "adaptive training procedure" [29]. "One-time training procedure" implies that the training process is computed over a comprehensive historical traffic dataset that incorporates all the possible situations to occur. In this manner the established model is comprehensive and can be used without additional training procedures. The adaptive training procedure implies repetitive trainings that are updated with the latest collection of data in order to setup the prediction model.

In addition to neural network-based approaches, many other machine learning techniques have been used for maritime traffic prediction's application. In [30], the authors proposed an associative learning-based approach that can predict vessels' navigation states like future position or velocity prediction [30]. Basically, associative learning is a type of data mining approaches that discovers taxonomic relationships between objects or concepts of interest [31].

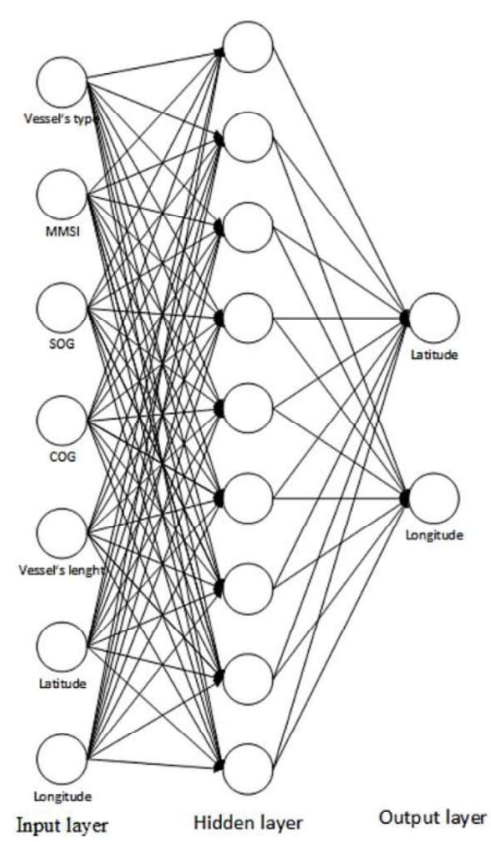

Figure 8. Model of Artificial Neural network for prediction of next turning region coordinates [25]

In the algorithm presented in [23], both location and velocity are discretized. Location discretization is based on uniform square grids over the area of interest and velocity is discretized as multiple speed bands with direction indication. 


\subsection{Image processing Method}

The visual recognition of traditional maritime targets is mainly based on digital image processing and pattern recognition methods. They are mainly being done through the following processes: image pretreatment, image smoothing and segmentation, feature extraction, and recognition through classifier [32].

In recent years, deep learning has developed rapidly and has widely been used. Neural networks based on deep learning can perform adaptive learning on available datasets, can extract useful information and can avoid artificial designs belonging to feature extraction operator. In recent years, deep convolution neural network has made an important progress in the fields of image classification and speech recognition [33]. It can directly use the original image as input, reduce the complexity of the network model and the number of weights and it is highly insensitive to visual deformation such as panning, scaling, and tilting [34], [35].

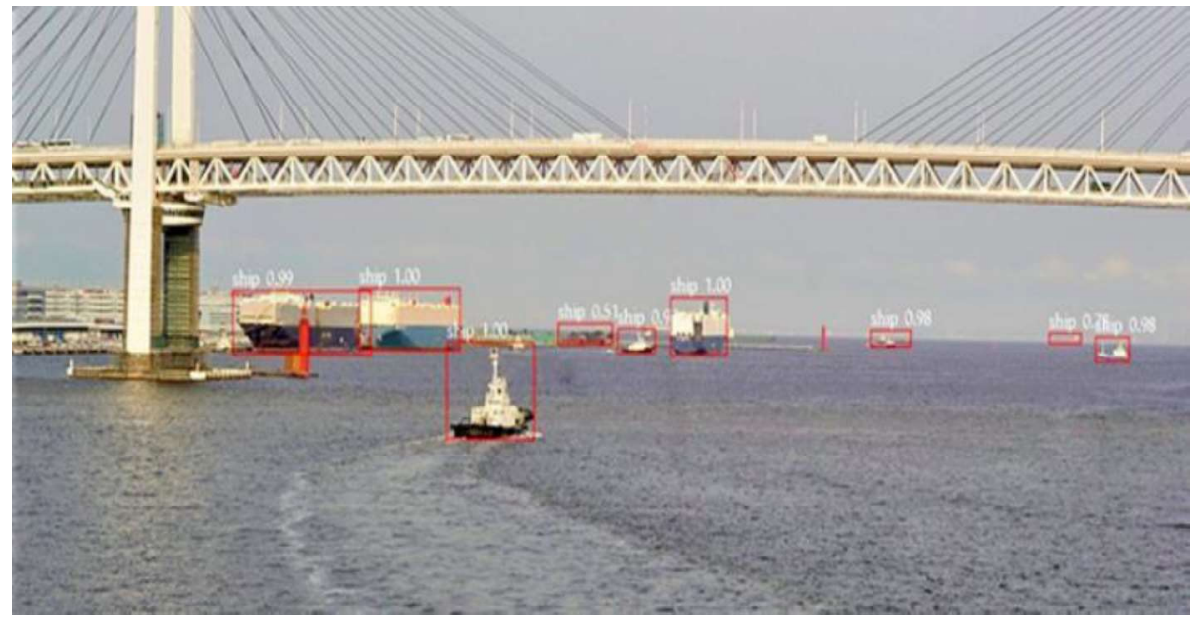

Figure 9. Vessels detection process from live imagery [36]

\section{4. "Defining Anomalous Behaviour" Approach}

Defining anomalous vessels patterns can have a starting point taking into consideration some elementary base facts such as:

- The majority of vessels, especially in open sea, seek to optimize fuel consumption by manoeuvring very seldom and by keeping their speed as constant.

- Vessels navigating in deep waters rarely perform manoeuvres in order to reduce ship trajectories into a sequence of waypoints [37].

- The international regulation requires AIS transponders to be installed aboard all ships of 300 gross tonnage and upwards engaged on international voyages, cargo ships of 500 gross tonnages and upwards not engaged on international voyages and all passenger ships irrespective of size.

Vessel behaviour can be represented as the sum of all parameters defining vessels movement, such as vessel type, position, speed, course, destination port or crew nationality, observed over a extended period of time. Vessel behaviour monitored over time, provides insights into the navigational patterns followed by each vessel on specific routes [3]. A vessel's pattern is made of events that tend to repeat in a specific timeframe and predictable manner. For example, while heading towards a fishing zone, it is normal for a fishing boat, but it should be marked as anomalous activity for a tug boat. Another example would be that low speed is normal for a cargo ships but not for speed vessels [38]. 
Although in recent years' vessel tracking data has become available for general purpose use, defining what normal vessel's behaviour is under specific temporal and spatial constraints, without the need of human expertise and interventions, has still remained a challenge.

In maritime surveillance and more specifically vessel motion, defining anomalous ship patterns can be categorized in some of the following manners [39]:

- Route Deviation: a vessel deviates from the route or path it should follow (deviation from the recommended route for Port destination sent by AIS transponder).

- Proximity: two or more vessels are abnormally close to each other indicating transfer of goods over sea.

- Parallel Waypoints: two or more vessels have parallel routes indicating illegal net fishing activities or military formations presence.

- Lost track or communication gap: a vessel intentionally hides its position and data by switching off its AIS transponder.

- Sailing in shallow waters: a vessel sails in shallow waters which indicates a possible grounding.

- Zero speed in non-anchorage areas: a vessel is in danger due to technical problems or it spills chemical/hazardous substances.

- AIS spoofing: two or more vessels transmit the same maritime mobile service identity (MMSI) over AIS channels in two different parts of the world.

The above methods rely on detecting kinematic anomalies that are related to location, course, speed, reporting and manoeuvre of vessels. A non-kinematic method would rely on suspicions related to static data like passengers, crew list, cargo list, last port of call and next port of call.

In the MS domain, these non-conforming patterns are referred as anomalies. Défense R\&D Canada [7] provides the following definition for the term anomaly in the context of the MS domain: "Something peculiar (odd, curious, weird, bizarre, atypical) because it is inconsistent with or deviating from what is usual, normal, or expected, or because it is not conforming to rules, laws or customs" [6].

\section{Conclusion}

Due to its deep implication in maritime security and safety, traffic analysis needs to be automatized. Nowadays, in many national agencies, this process is still based only on human expertise and knowledge for analysing naval traffic behaviours. The main drawback of this kind of approach is its absolute reliance on human expertise for supplying traffic rules or labels, labels that may be inaccurate, incomplete or biased toward subjective motivations. A combined method of humans and machine is needed. Human ability for observation and reasoning from its experience represent a great advantage which is still irreplaceable by any computed based solution. Processes like forecasting, risk evaluation or mitigation tasks normally need to be conducted for a large number of monitored vessels. Taking into consideration the stream processing that has timeliness requirement at the system level the computing concurrency is an important consideration.

Since maritime traffic surveillance rely heavily on big scale data, pattern recognitions, route discoveries and building adequate models imply matching processing capabilities that can handle enormous scale data. As presented in this paper, most of the algorithms come at the expense of great complexity. In order to fulfil most of the requirements needed for real time maritime surveillance, it is relevant to establish efficient computing solutions to fully exploit the model complexity of these advanced AI technologies. 


\section{References}

[1] V. Fernandez Arguedas, G. Pallotta and M. Vespe, "Maritime Traffic Networks: From Historical Positioning Data to Unsupervised Maritime Traffic Monitoring," in IEEE Transactions on Intelligent Transportation Systems, vol. 19, no. 3, pp. 722-732, March 2018, doi: 10.1109/TITS.2017.2699635.

[2] NSPD 41/HSPD13, National Plan To Achieve Maritime Domain Awareness For The National Strategy For Maritime Security, 2005.

[3] Dimitrios Zissis, Elias K. Xidias, Dimitrios Lekkas, A cloud based architecture capable of perceiving and predicting multiple vessel behaviour, Applied Soft Computing, Volume 35, 2015, Pages 652-661, ISSN 1568-4946, https://doi.org/10.1016/j.asoc.2015.07.002.

[4] Ewa Osekowska, Henric Johnson, Bengt Carlsson, Grid Size Optimization for Potential Field based Maritime Anomaly Detection, Transportation Research Procedia, Volume 3, 2014, Pages 720729, ISSN 2352-1465, https://doi.org/10.1016/j.trpro.2014.10.051.

[5] I.H. Witten, E. Frank, Data Mining: Practical Machine Learning Tools and Techniques, Second Edition, Morgan Kaufmann Publishers (Elsevier), 2005.

[6] Samira Kazemi, Shahrooz Abghari, Niklas Lavesson, Henric Johnson, Peter Ryman, Open data for anomaly detection in maritime surveillance, Expert Systems with Applications, Volume 40, Issue 14, 2013, Pages 5719-5729, ISSN 0957-4174, https://doi.org/10.1016/j.eswa.2013.04.029.

[7] Roy, J. (2008). Anomaly detection in the maritime domain. Proceedings of SPIE - The International Society for Optical Engineering, USA, 6945, 69450W-1-69450W-14. doi:10.1117/12.776230

[8] A. ITANI, C. RAY, A. El Falou and J. Issa, "Mining Ship Motions and Patterns of Life for the EU Common Information Sharing Environment (CISE)," OCEANS 2019 - Marseille, Marseille, France, 2019, pp. 1-6, doi: 10.1109/OCEANSE.2019.8867219.

[9] K. Dästner, B. von Haßler zu Roseneckh-Köhler, F. Opitz, M. Rottmaier and E. Schmid, "Machine Learning Techniques for Enhancing Maritime Surveillance Based on GMTI Radar and AIS," 2018 19th International Radar Symposium (IRS), Bonn, 2018, pp. 1-10, doi: 10.23919/IRS.2018.8447961.

[10] Steven Mascaro, Ann E. Nicholso, Kevin B. Korb, Anomaly detection in vessel tracks using Bayesian networks, International Journal of Approximate Reasoning, Volume 55, Issue 1, Part 1, 2014, Pages 84-98, ISSN 0888-613X, https://doi.org/10.1016/j.ijar.2013.03.012.

[11] T. Mantecón, D. Casals, J. J. Navarro-Corcuera, C. R. del-Blanco and F. Jaureguizar, "Deep Learning to Enhance Maritime Situation Awareness," 2019 20th International Radar Symposium (IRS), Ulm, Germany, 2019, pp. 1-8, doi: 10.23919/IRS.2019.8768142.

[12] Michele Fiorini, Andrea Capata, Domenico D. Bloisi, AIS Data Visualization for Maritime Spatial Planning (MSP), International Journal of e-Navigation and Maritime Economy, Volume 5, 2016, Pages 45-60, ISSN 2405-5352, https://doi.org/10.1016/j.enavi.2016.12.004.

[13] C. Zor and J. Kittler, "Maritime anomaly detection in ferry tracks," 2017 IEEE International Conference on Acoustics, Speech and Signal Processing (ICASSP), New Orleans, LA, 2017, pp. 2647-2651, doi: 10.1109/ICASSP.2017.7952636.

[14] C. Leys, C. Ley, O. Klein, P. Bernard, and L. Licata, "Detecting outliers: Do not use standard deviation around the mean, use absolute deviation around the median," Journal of Experimental Social Psychology, vol. 49, no. 4, pp. 764 - 766,2013.

[15] Angelos Valsamis, Konstantinos Tserpes, Dimitrios Zissis, Dimosthenis Anagnostopoulos, Theodora Varvarigou, Employing traditional machine learning algorithms for big data streams analysis: The case of object trajectory prediction, Journal of Systems and Software, Volume 127, 2017, Pages 249-257, ISSN 0164-1212, https://doi.org/10.1016/j.jss.2016.06.016.

[16] Y.-Y. Su and S.-J. Chang, "Spatial cluster detection for the fishing vessel monitoring systems," in Proc. OCEANS, Apr. 2008, pp. 1-4.

[17] S.-J. Chang, G.-Y. Hsu, J.-A. Yang, K.-N. Chen, Y.-F. Chiu, and F.-T. Chang, "Vessel traffic analysis for maritime intelligent transportation system," in Proc. IEEE Veh. Technol. Conf., May 
2010, pp. 1-4.

[18] M. Y. Santos, J. P. Silva, J. Moura-Pires, and M. Wachowicz, "Automated traffic route identification through the shared nearest neighbour algorithm," in Bridging the Geographic Information Sciences, Berlin, Germanty: Springer, 2012, pp. 231-248.

[19] G. Pallotta, M. Vespe, and K. Bryan, "Vessel pattern knowledge discovery from AIS data: A framework for anomaly detection and route prediction," Entropy, vol. 15, no. 6, pp. 2218-2245, 2013.

[20] V. F. Arguedas, G. Pallotta, and M. Vespe, "Automatic generation of geographical networks for maritime traffic surveillance," in Proc. Int. Conf. Inf. Fusion (FUSION), Jul. 2014, pp. 1-8.

[21] B. Ristic, B. La Scala, M. Morelande, and N. Gordon, "Statistical analysis of motion patterns in AIS data: Anomaly detection and motion prediction," in Proc. 11th Int. Conf. Inf. Fusion, Jun./Jul. 2008, pp. 1-7.

[22] R. Wen, W. Yan, A. N. Zhang, N. Q. Chinh, and O. Akcan, "Spatiotemporal route mining and visualization for busy waterways," in Proc. IEEE Int. Conf. Syst., Man, Cybern. (SMC), Oct. 2016, pp. 849-854.

[23] Z. Xiao, X. Fu, L. Zhang and R. S. M. Goh, "Traffic Pattern Mining and Forecasting Technologies in Maritime Traffic Service Networks: A Comprehensive Survey," in IEEE Transactions on Intelligent Transportation Systems, vol. 21, no. 5, pp. 1796-1825, May 2020, doi: 10.1109/TITS.2019.2908191.

[24] Heaton, J. (2015). Artificial Intelligence for Humans Volume 3: Deep Learning and Neural Networks. Heaton Research, Inc.

[25] A. Daranda, "Neural network approach to predict marine traffic," Balt. J. Mod. Comput., vol. 4, no. 3, pp. 483-495, 2016.

[26] B. L. Young, "Predicting vessel trajectories from AIS data using R," M.S. thesis, Naval Postgraduate School, Monterey, CA, USA, 2017.

[27] A. Khan, C. Bil, and K. E. Marion, "Theory and application of artificial neural networks for the real time prediction of ship motion," in Proc. Int. Conf. Knowl.-Based Intell. Inf. Eng. Syst., vol. 1, 2005, pp. 1064-1069.

[28] T. Xu, X. Liu, and X. Yang, "A novel approach for ship trajectory online prediction using BP neural network algorithm,” Int. J. Adv. Inf. Sci. Service Sci., vol. 4, no. 11, pp. 271-277, 2012.

[29] P. Borkowski, "The ship movement trajectory prediction algorithm using navigational data fusion," Sensors, vol. 17, no. 6, p. 1432, 2017.

[30] N. A. Bomberger, B. J. Rhodes, M. Seibert, and A. M. Waxman,

"Associative learning of vessel motion patterns for maritime situation awareness," in Proc. 9th Int. Conf. Inf. Fusion, Jul. 2006, pp. 1-8.

[31] B. J. Rhodes, "Taxonomic knowledge structure discovery from imagery-based data using the neural associative incremental learning (NAIL) algorithm," Inf. Fusion, vol. 8, no. 3, pp. 295-315, 2007.

[32] Bayramoglu N, Alatan A A. Shape Index SIFT: Range Image Recognition Using Local Features[C]// International Conference on Pattern Recognition. IEEE Computer Society, 2010:352355.

[33] J. K. Chorowski, D. Bahdanau, D. Serdyuk, K. Cho, and Y. Bengio. Attention-based models for Processing Systems (NIPS), 2015

[34] K. Simonyan and A. Zisserman. Very deep convolutional networks for large-scale image recognition. In ICLR, 2015.

[35] H. Fu, Y. Li, Y. Wang and L. Han, "Maritime Target Detection Method Based on Deep Learning," 2018 IEEE International Conference on Mechatronics and Automation (ICMA), Changchun, 2018, pp. 878-883, doi: 10.1109/ICMA.2018.8484727.

[36] https://www.ship-technology.com/news/vessel-image-recognition-mol

[37] N. Forti, L. M. Millefiori and P. Braca, "Unsupervised extraction of maritime patterns of life from Automatic Identification System data," OCEANS 2019 - Marseille, Marseille, France, 2019, pp. 1-5, doi: 10.1109/OCEANSE.2019.8867429. 
[38] K. Kowalska and L. Peel, "Maritime anomaly detection using Gaussian Process active learning," 2012 15th International Conference on Information Fusion, Singapore, 2012, pp. 11641171.

[39] K. Chatzikokolakis, D. Zissis, M. Vodas, G. Spiliopoulos and I. Kontopoulos, "A distributed lightning fast maritime anomaly detection service," OCEANS 2019 - Marseille, Marseille, France, 2019, pp. 1-8, doi: 10.1109/OCEANSE.2019.8867269.

[40] Ewa Osekowska, Henric Johnson, Bengt Carlsson, Maritime vessel traffic modeling in the context of concept drift, Transportation Research Procedia, Volume 25, 2017, Pages 1457-1476, ISSN 2352-1465, https://doi.org/10.1016/j.trpro.2017.05.173.

[41] X. Wu, A. Rahman, V. A. Zaloom, and B. N. Craig, "Study of vessel travel behavior at hot spots in Sabine-Neches waterway," in Proc. Transp. Res. Board 96th Annu. Meeting, 2017, pp. 1-17.

[42] https://en.wikipedia.org/wiki/Artificial_neural_network 